\title{
Detection of gratings and small features in speckle imagery
}

\author{
Vijaya N. Korwar and John R. Pierce
}

\begin{abstract}
The extent of picture degradation of speckle, in particular in synthetic aperture radar pictures, has been investigated in the cases where an observer has to detect (a) a small feature immersed in a darker background, and (b) a square wave grating. In each case, a theoretical model is developed for the observer's detection mechanism, and the probability of correct decision is related to relevant picture parameters such as contrast, looks per pixel, and size. These calculations are verified by psychophysical experiments using computersimulated pictures. Detectability of gratings as a criterion for characterizing picture quality is shown to be far inferior to feature detectability.
\end{abstract}

\section{Introduction}

The phenomenon of speckle ${ }^{1-3}$ in coherent systems and the degradation of picture quality caused by speckle ${ }^{4-6}$ have long been known. The work described here was motivated by the problem of picture degradation by speckle in synthetic aperture radar (SAR) systems, ${ }^{7-9}$ which are coherent high-resolution radar systems used for terrain mapping; however, the results are applicable to other systems where speckle obscures desired detail in pictures. In particular, recent and proposed SAR systems, such as those in the JPL missions SEASAT, VOIR, SIR-A, will provide oceanographers and geologists with land and ocean maps. In all these cases, the pictures are viewed by a human observer. It is necessary to be able to relate the probability that the observer correctly detects various features, such as craters in the speckled picture, to the parameters of the picture and to be able to design the system to meet suitable criteria for tolerable picture quality. Possible criteria are the detectability of squares and line gratings, the latter being commonly used in TV pictures. This paper reports the investigation of these two problems theoretically and by psychophysical experiments and compares the two criteria.

Dainty ${ }^{4}$ has investigated theoretically the problem of detecting small objects by a machine that uses a

Both authors are with California Institute of Technology, Pasadena; V. N. Korwar is in the Jet Propulsion Laboratory, California 91103, and J. R. Pierce is in the Electrical Engineering Department, California 91125 .

Received 24 June 1980.

0003-6935/81/020312-08\$00.50/0.

(C) 1981 Optical Society of America.
Neyman-Pearson type of decision strategy. Since a human observer's decision strategy is not fully understood, we need to make reasonable assumptions about it in connection with our problem. The decision strategy we use and the details of the problem we are concerned with differ from Dainty's. George et al. ${ }^{5}$ have considered the problem of detecting small objects, both theoretically and experimentally, but again, with an approach and emphasis different from ours. Kozma and Christensen ${ }^{6}$ have considered experimentally the detection of gratings in speckle, and their approach and emphasis also differ from ours.

\section{Picture Parameters}

It is well known that speckle effects decrease with an increase in the number of looks $L$, i.e., the number of independent estimates of each pixel intensity that are averaged to form a pixel in the final picture. These looks are obtained in SAR systems ${ }^{9}$ by various techniques such as the use of different carrier frequencies, aspect angles, or polarizations. Each of these techniques increases the complexity of a digital SAR processor required to achieve a given resolution cell size.

We will restrict our attention to pictures that, in the absence of speckle, can be described by two levels of intensity. We denote the fractional difference in intensity of these two levels by $b$. Thus, in the small feature detection problem, the feature and background pixels have intensities $P_{0}(1+b)$ and $P_{0}$, respectively; in the grating detection problem, the pixels in the alternating bright and dark lines have intensities $P_{0}(1+$ $b$ ) and $P_{0}$, respectively. The factor $(1+b)$ will be called the contrast ratio.

Experiments showed that the absolute intensity $P_{0}$ does not noticeably influence detectability, provided the brightness corresponding to $P_{0}$ lies well within the range of brightness sensitivity of the human visual system. Hence $P_{0}$ will not figure in our results and is 
only defined for the sake of generality of analysis. However, it is clear that increasing the contrast ratio (1 $+b$ ) between a feature intensity and that of its surroundings should increase the feature detectability. To see why this is an important parameter, consider the processing of raw SAR data to produce an image. This involves a 2-D matched filtering of the signal returns, ${ }^{7}$ and the autocorrelation function (acf) of the filter impulse response is not, in practice, the ideal $\delta$ function. An increase in the integrated side-lobe ratio (ISLR) of this impulse response reduces the contrast between a feature and its surroundings. Reducing the ISLR while meeting other SAR specifications requires careful system design.

These considerations show the desirability of performing a trade off between $L$ and $(1+b)$ required to achieve a desired picture quality defined in some suitable way.

Other parameters, which we will call the geometrical parameters, that can affect the detectability of a small square are the picture size $W \times W$ and the size of the square $D \times D$. All our specifications of size will be in pixels. Similarly, the detectability of a grating of height $V$, total width $H$, and $D$ pixels per line is expected to depend on $H, V, D$.

Although we consider specifically (in the following section) the detection of small $D \times D$ squares, these results are applicable to the detection of any small feature of area $D^{2}$.

\section{Detection of Small Features $(D \ll W)$}

\section{A. Detection Model}

Our detection model is based on the following assumptions:

(a) The first assumption is that the viewer's visual system responds to the total optical power reflected by a stimulus, provided the stimulus subtends a small enough angle at the eye ( $\$ 10 \mathrm{~min}$ of arc). This has, in fact, been demonstrated by various workers using psychophysical and neurological techniques to be true under various conditions. ${ }^{10-13}$ This assumption is also supported by George et al. ${ }^{5}$

(b) The second assumption is that the observer looks at the total power from every $D \times D$ square in the picture and chooses the brightest one. Since the observer knows what the value of $D$ is, this is plausible. This is what one form of ideal detector would do. The assumption of the human observer being an ideal detector for visual signals in noise has been made before in work on vision and shown to be reasonable. ${ }^{14-16}$

Further justification for the use of this model is given elsewhere. ${ }^{17}$

\section{B. Theoretical Calculations}

\section{Basic Equations}

First, we note that there are $N=W^{2}$ possible $D \times D$ squares in the background, neglecting edge effects. If we consider the total power reflected by each of these. $W^{2}$ squares, we have $W^{2}$ random variables of which only
$W^{2} / D^{2}$ are independent. However, for the case of large $N=W^{2}$ and probability $p_{c}$ of correct detection $\gtrsim 0.5$, we show that the interdependence of these $N$ background powers makes little difference to the results calculated assuming independence. We assume this independence even for the case of $p_{c}<0.5$, and the results in this region are approximate to this extent. In any case, the results are not very sensitive to the exact value of large $N$.

The probability $p_{c}$ of correct detection is the probability that the feature power, which we denote by $G$, is actually greater than the largest of the $N$ background powers denoted by $B_{i}$. The $G$ and $B_{i}$ are known ${ }^{18}$ to be multiples of random variables having a $\gamma$ density function with parameter $M=L D^{2}$ or of $\chi^{2}$ distributed random variables with $2 M$ degrees of freedom. In terms of the probability density functions (pdfs) of the $G$ and $B_{i}, p_{G}(x)$, and $p_{B}(x)$, we have

$$
\begin{aligned}
p_{c} & =\int_{0}^{\infty} p_{G}(x) A_{N}(x) d x \\
& =\int_{0}^{\infty} p_{G}(x)\left[\int_{0}^{x} p_{B}(y) d y\right]^{N} d x,
\end{aligned}
$$

where

$$
\begin{aligned}
& p_{B}(x)=\frac{1}{P_{0}^{M}} x^{(M-1)} \frac{\exp \left(-x / P_{0}\right)}{\Gamma(M)} \text { for } x>0, \\
& p_{G}(x)=\frac{1}{P_{0}^{M}(1+b)^{M}} \frac{x^{(M-1)}}{\Gamma(M)} \exp \left\{-x /\left[P_{0}(1+b)\right]\right\} \text { for } x>0,
\end{aligned}
$$

$A_{N}(x)=P$ [largest of $\left.B_{i}(i=1,2, \ldots, N) \leq x\right]$.

$p_{B}(x)$ and $p_{G}(x)$ are zero for $x<0$.

Equation (1) cannot be evaluated in closed form, and even numerical evaluation is complicated by convergence problems because of the large value of $N$, especially for the case of large $M$, where the variance of $G$ and $B_{i}$ is appreciable. To simplify this evaluation, we use the asymptotic distribution ${ }^{19}$ of the largest value of the $B_{i}$, which we verified by direct calculation to be approximately valid for the region where $A_{N}(x)>0.05$. Thus for $p_{c}>0.5$, this asymptotic expression $A_{N a}(x)$ can be used in Eq. (1) with $\sim 10 \%$ error or less. The asymptotic expression is

$$
A_{N a}(x)=\exp \left\{-\exp \left[-a_{N}\left(x-x_{N}\right)\right]\right\},
$$

where $x_{N}=$ characteristic largest value of a sample of $N$ variables $B_{i}$ and $a_{N}=$ intensity function evaluated at $x=x_{N}$.

These can be defined by

$$
\begin{gathered}
F\left(x_{N}\right)=1-(1 / N), \\
a_{N}=N f\left(x_{N}\right),
\end{gathered}
$$

where $f(x)$ and $F(x)$ are the pdf and distribution function of any one of the random variables $B_{i}$. tion

We then have, in place of Eq. (1), the approxima-

$$
p_{c}=\int_{0}^{\infty} p_{G}(x) A_{N a}(x) d x .
$$




\begin{tabular}{|c|c|c|c|c|c|c|c|c|}
\hline \multirow[b]{2}{*}{$(1+b) \mathrm{dB}$} & \multirow[b]{2}{*}{$L$} & \multirow[b]{2}{*}{$D$} & \multirow[b]{2}{*}{$M$} & \multirow{2}{*}{$\begin{array}{c}\text { Number of } \\
\text { observations } \\
n\end{array}$} & \multirow[b]{2}{*}{$\begin{array}{c}\text { Theoretical } \\
p_{c}\end{array}$} & \multicolumn{3}{|c|}{ Experimental $\hat{p}_{c}$} \\
\hline & & & & & & $\begin{array}{l}\text { Lower } \\
\text { bound }\end{array}$ & $\hat{p}_{c}$ & $\begin{array}{l}\text { Upper } \\
\text { bound }\end{array}$ \\
\hline 1 & $\begin{array}{l}14 \\
10 \\
18\end{array}$ & $\begin{array}{l}6 \\
8 \\
6\end{array}$ & $\begin{array}{l}504 \\
640 \\
648\end{array}$ & $\begin{array}{l}48 \\
60 \\
84\end{array}$ & $\begin{array}{r}0.65 \\
0.95 \\
>0.95\end{array}$ & $\begin{array}{l}0.37 \\
0.71 \\
0.85\end{array}$ & $\begin{array}{l}0.52 \\
0.82 \\
0.93\end{array}$ & $\begin{array}{l}0.67 \\
0.93 \\
0.98\end{array}$ \\
\hline 3 & $\begin{array}{l}1 \\
1 \\
9 \\
7 \\
2 \\
1\end{array}$ & $\begin{array}{r}5 \\
6 \\
2 \\
3 \\
6 \\
10\end{array}$ & $\begin{array}{r}25 \\
36 \\
36 \\
63 \\
72 \\
100\end{array}$ & $\begin{array}{l}60 \\
48 \\
24 \\
12 \\
12 \\
48\end{array}$ & $\begin{array}{r}0.63 \\
0.72 \\
0.72 \\
0.95 \\
>0.95 \\
>0.95\end{array}$ & $\begin{array}{l}0.20 \\
0.52 \\
0.33 \\
0.64 \\
0.80 \\
0.93\end{array}$ & $\begin{array}{l}0.32 \\
0.67 \\
0.54 \\
0.92 \\
0.92 \\
1.0\end{array}$ & $\begin{array}{l}0.45 \\
0.80 \\
0.74 \\
1.0 \\
1.0 \\
1.0\end{array}$ \\
\hline 5 & $\begin{array}{r}1 \\
2 \\
1 \\
1 \\
22 \\
1 \\
1 \\
1\end{array}$ & $\begin{array}{l}2 \\
2 \\
3 \\
4 \\
1 \\
5 \\
6 \\
7\end{array}$ & $\begin{array}{r}4 \\
8 \\
9 \\
16 \\
22 \\
25 \\
36 \\
49\end{array}$ & $\begin{array}{l}48 \\
24 \\
60 \\
60 \\
24 \\
48 \\
60 \\
48\end{array}$ & $\begin{array}{l}0.24 \\
0.52 \\
0.59 \\
0.85 \\
0.96 \\
0.98 \\
0.999 \\
0.9999\end{array}$ & $\begin{array}{l}0.05 \\
0.37 \\
0.14 \\
0.59 \\
0.63 \\
0.72 \\
0.88 \\
0.93\end{array}$ & $\begin{array}{l}0.13 \\
0.58 \\
0.24 \\
0.72 \\
0.83 \\
0.85 \\
0.97 \\
1.0\end{array}$ & $\begin{array}{l}0.25 \\
0.78 \\
0.36 \\
0.82 \\
0.96 \\
0.94 \\
1.0 \\
1.0\end{array}$ \\
\hline 8 & $\begin{array}{l}8 \\
1\end{array}$ & $\begin{array}{l}1 \\
3\end{array}$ & $\begin{array}{l}8 \\
9\end{array}$ & $\begin{array}{l}24 \\
12\end{array}$ & $\begin{array}{l}0.95 \\
0.95\end{array}$ & $\begin{array}{l}0.63 \\
0.77\end{array}$ & $\begin{array}{l}0.83 \\
1.0\end{array}$ & $\begin{array}{l}0.96 \\
1.0\end{array}$ \\
\hline
\end{tabular}

The results of the theoretical calculation of $p_{c}$ for various parameters are shown in Table I; the results for the case $p_{c} \leq 0.5$ shown are obtained by using Eq. (1) directly, the rest by using Eq. (6).

The $N$ variables $B_{i}$ have a short-range correlation, i.e., the correlation of each $B_{i}$ is only with $D^{2}$ of the $N$ variables $B_{i}$. In such a case, for certain distributions of the $B_{i},{ }^{20}$ of which the present distribution can be shown to be one, the asymptotic distribution of the largest value is the same as in the case of independence, i.e., Eq. (3).

Thus, for the range $p_{c}>0.5$, where we can use the asymptotic distribution, the interdependence of the $N$ background powers $B_{i}$ can be neglected.

\section{Further Approximations}

Equation (1) is not very useful for design purposes, i.e., for purposes of interrelating the parameters $b, D$, $W, L$ required to produce a desired threshold probability $p_{c}=p_{t}$, because this would involve a trial-anderror solution of Eq. (1).

For this purpose, we further simplify Eq. (6) to get ${ }^{17}$ the following relationship:

$$
\frac{z_{p l}(N)+(4 M-1)^{1 / 2}}{(1+b)^{1 / 2}}-(4 M-1)^{1 / 2}=z_{p t},
$$

where $z_{p l}(N), z_{p t}$ are $100 p_{l}$ and $100 p_{t}$ percentage points of the normal distribution,

$$
\begin{aligned}
& p_{l}=(0.95)^{1 / N}, \\
& M=L D^{2} .
\end{aligned}
$$

This equation gives the desired relationship between the parameters $b, L, D, W$ at threshold, where threshold means here the point at which $p_{c}$ equals some specified value $p_{t}$. We will refer to the corresponding values of $b, L, D, W$ as threshold values. For $b \ll 1$, a further approximation leads to

$$
b D \sqrt{L} \approx \text { constant, }
$$

which shows that, for low contrast ratios, an increase in $L$ is less effective than an increase in contrast to achieve a threshold $p_{t}$.

We will now outline the derivation of Eq. (7). First, note that the function $A_{N a}(x)$, for large $N$, has a stepfunctionlike appearance with the step occurring near the characteristic largest value $x_{N}$. In Fig. 1, we plot a specific example of the pdfs of the feature and background powers and the asymptote $A_{N a}(x)$. We see from Eq. (6) that any appreciable value $p_{c}$ has must be because the peak of the density function $p_{G}(x)$ occurs at a value of $x$ close to $x_{N}$ or greater than $x_{N}$. For this reason, we can get an approximate value of $p_{c}$ for large $p_{c}$ by setting $p_{c}$ approximately equal to the integral of the $p_{G}(x)$ curve from $x_{1}$ to $\infty$, where $x_{1}$ is, say, the point where $A_{N}(x) \simeq 0.95$. This is defined by the $100 p_{l} \%$ point of the $\chi^{2}$ distribution with $2 \mathrm{M}$ degrees of freedom,

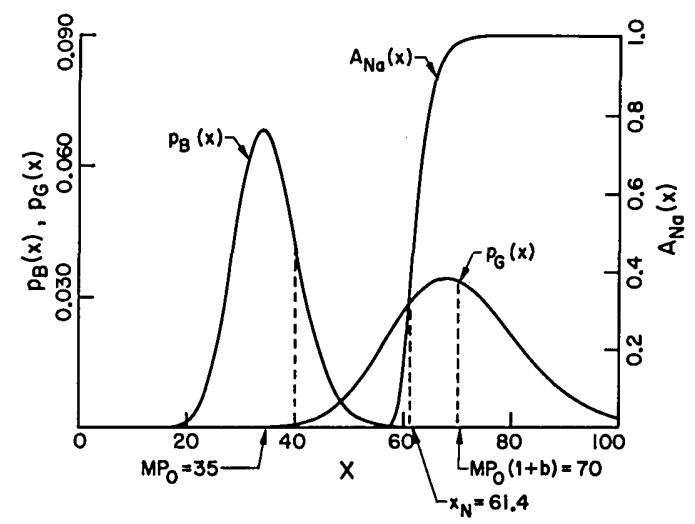

Fig. 1. Probability density functions $p_{B}(x), p_{G}(x)$ with means $M P_{0}$ and $M P_{0}(1+b)$ and asymptotic distribution $A_{N a}(x)$ of largest of $N$ variables $B_{i}$ with characteristic largest value $x_{N}$, all with $N=10^{4}, M$ $=35, b=1$, and $P_{0}=1$. 
Table II. Predicted Threshold Parameters for $p_{t}=0.95, N=10^{4}$

\begin{tabular}{crc}
\hline$(1+b) \mathrm{dB}$ & $M$ & $D$ for $L=12$ \\
\hline 1 & 641 & 7.3 \\
3 & 65 & 2.3 \\
5 & 22 & 1.4 \\
8 & 8 & 0.8 \\
\hline
\end{tabular}

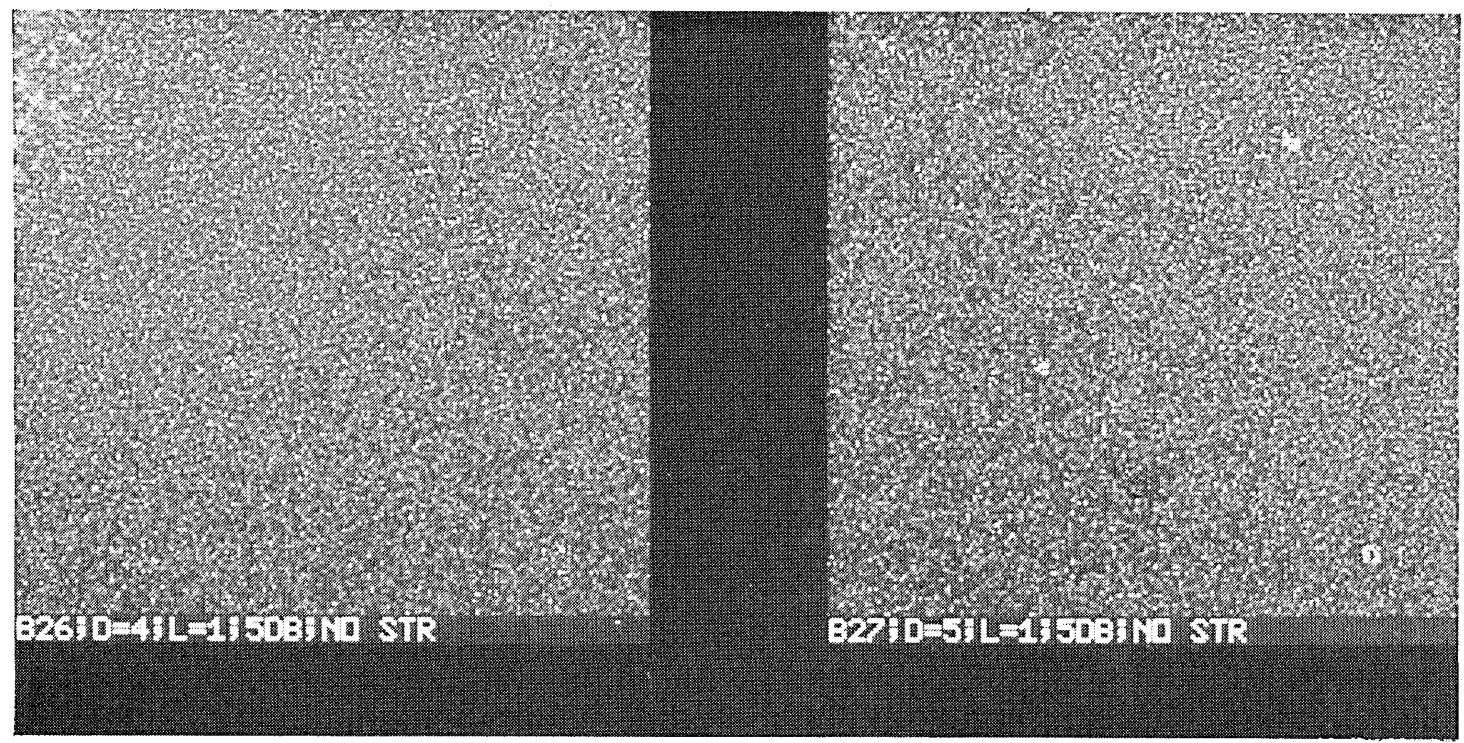

Fig. 2. Detection of squares, examples of simulated pictures. Both photographs have $L=1$ and 5 -dB constrast: (left) $D=4$; (right) $D=5$.

with $p_{l}=(0.95)^{1 / N}$. By using the Gaussian approximation to the $\sqrt{2 \chi^{2}}$ distribution function, which is valid for $M>50,{ }^{21}$ we can relate this to the $100 p_{l} \%$ point of the normal distribution. We assume that the same formula holds approximately for $M<50$ as well. Working through the details, we obtain the desired Eq. (7). We can show, for $p_{c}>0.9$, that the error in using this method to calculate $p_{c}$ is at most 0.1 and decreases as $p_{c}$ increases.

Calculations básed on Eq. (7) are given in Table II.

\section{Simulations and Experiments}

A large number of pictures were generated by computer for various values of the parameters $b, L, D, W$ and photographed with an on-line image generator (Dichomed). Each pixel in the speckle-corrupted picture was generated by averaging the squares of $2 L$ independent, identically distributed Gaussian pseudorandom variables with variances proportional to the intensity $P_{0}$ or $P_{0}(1+b)$ of the corresponding unspeckled pixel. The justification for this technique is given by Guenther et al., ${ }^{22}$ who use it in simulating fully developed speckle passed through a linear filter whose transfer function represents temporal averaging.

Each picture was a $200 \times 200$ picture, in which each quadrant contained a single $D \times D$ bright square at a random location. By masking all but one quadrant of the picture, only a $100 \times 100$ picture containing one bright $D \times D$ square was shown to the observer each time. The observer was initially shown an unspeckled picture (with a different location of the square) to allow him to judge what size square he was looking for; moreover, this is a psychophysical technique that has the same effect as a training period. ${ }^{14}$ The observer's location of the square in the speckled picture was compared with its actual location, and the probability of correct detection was estimated by $\hat{p}_{c}=$ the fraction of correct responses made by the observer. Some examples of the simulated pictures are shown in Fig. 2. This figure demonstrates the validity of the predicted threshold parameters for the 5-dB case. The photograph on the left has $L=1, D=4$, so that $M=16$, while the other one has $L=1, D=5$, so that $M=25$, which is the predicted threshold for $p_{c}=0.95$ (Table II). The four squares in the $M=25$ picture are clearly visible, while the ones in the $M=16$ picture, whose locations have been chosen to be the same as in the $M=25$ case, are not clear.

The results of the experiments are shown along with the theoretical calculations in Table I. Most of the parameter sets were chosen to be near the predicted threshold values, because our main interest in the experiments was to verify the predicted threshold relationship. The theoretical and experimental probabilities of error, denoted by $p_{e}=1-p_{c}$ and $\hat{p}_{e}=1-\hat{p}_{c}$, 


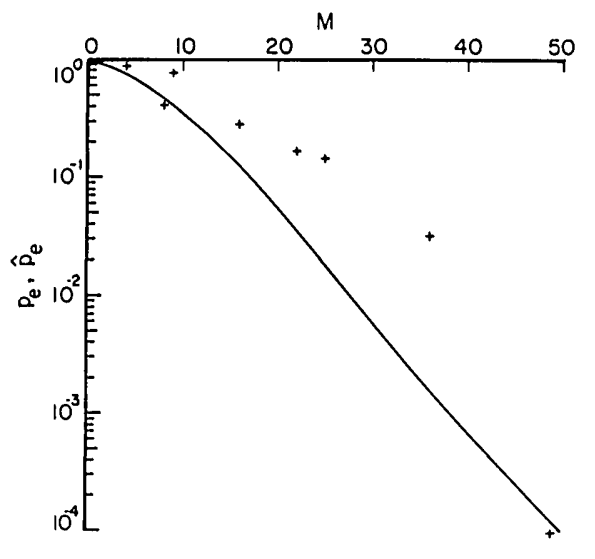

Fig. 3. Theoretical and experimental probabilities of error $p_{e}$ (continuous curve) and $\hat{p}_{e}$ (discrete points) vs $M$ for $N=10^{4}, b=$ 2.16 .

respectively, are plotted in Fig. 3 for this 5 -dB case.

The upper and lower bounds on $p_{c}$ given in all the tables are $95 \%$ confidence limits, obtained by the standard statistical method ${ }^{23}$ for estimating proportions in a sample of size $n$.

\section{Conclusions for Feature Detection}

From Tables I and II and Fig. 3, we see that

(1) usually $\hat{p}_{c}<p_{c}$, but both follow the same sort of increase with $b$ and $M$;

(2) the difference between $p_{c}$ and $\hat{p}_{c}$ is small for $p_{c}$ $\geqslant 0.9$;

(3) the threshold parameter values predicted by Eq.

(7) are close to the experimental ones.

In general, we conclude that the experimental results are in agreement with the theoretical model developed, although more experiments would be required to establish it as the correct one.

\section{Grating Detection}

\section{A. General Method}

We consider the problem of obtaining the probability $p_{c}$ of detecting correctly whether a given $H \times V$ speckled $L$-look picture contains a square-wave grating with lines $D$ pixels wide and alternating pixel intensities $P_{0}$ and $P_{0}(1+b)$. The technique used here relies more on experiment than did the technique of the previous problem because of the greater complexity of this problem. We develop a reasonable model for grating detection and define a signal-to-speckle-noise ratio (SSNR) that incorporates all the parameters $b, L, H, V$, $D$ and which uniquely defines $p_{c}$. We then obtain $p_{c}$ experimentally from a number of experiments much larger than in the previous problem and find the empirical relationship between $p_{c}$ and SSNR. This relationship can then be used to get $p_{c}$ for any given set of parameters $b, L, D, H, V$. All gratings considered have lines running vertically.

\section{B. Detection Model}

Our detection model here is based on the following assumptions:

(a) First, we assume that the spatial distribution of intensity reflected by the picture is passed through the observer's visual filter centered on the (fundamental) frequency $f_{g}$ of the grating that the observer is looking for; this filter has a flat bandpass over \pm 1 octave, i.e., from $0.5 f_{g}$ to $2 f_{g}$. Previous work ${ }^{24-26}$ has shown that there exist, in the visual nervous system, several visual channels, each tuned to a different limited range of spatial frequencies and each extending \pm 1 octave about its central frequency. The assumption that the filter centered on $f_{g}$ is relevant even when there is no grating present is justified by the fact that the observer is told what $f_{g}$ he is looking for, so that his brain considers only the output of that particular filter that is tuned to $f_{g}$.

(b) Second, we assume that either the mean or the peak of the amplitude of the envelope of the filter output in each correlation interval of the output is compared with some threshold level to obtain one estimate of the decision. If the threshold is exceeded, a signal (grating) is said to be present; if not, the decision is that the input is just noise. This is based on ideal detector theory.

(c) The various possible estimates of decision from an $H \times V$ grating are assumed to be combined in some way to reach a decision. We make the assumption that each piece of the grating of height $V=1$ pixel and horizontal extent $H=2 D / 1.5=$ the correlation length of the filter output gives rise to one estimate. Thus, there are $N=H V /(2 D / 1.5)$ possible estimates in an $H \times V$ grating. This may be wrong by a scale factor $a_{H} a_{V}$, but we are not interested in an absolute SSNR; we are interested only in relating the various parameters to $p_{c}$. Thus if $a_{H} a_{V}$ is constant, independent of $H, V$, which is equivalent to the assumption that the observer maintains his detection criteria from one experiment to the next, we may set $a_{H} a_{V}$ equal to 1 . This assumption of the observer's consistency is usually reasonable, as has been shown before. ${ }^{14}$

\section{Obtaining the SSNR Expression}

We approximate the $\gamma$ pdf of each pixel intensity by a Gaussian pdf with mean $L P$ and variance $L P^{2}$, where $P$ is $P_{0}$ or $P_{0}(1+b)$ for the dark and bright lines, respectively. This approximation is valid only for large $L$, but we extend the SSNR based on this assumption to the case of arbitrary $L$.

To derive the desired SSNR expression, we first consider the partial $\mathrm{SSNR}_{p}$ in one estimate of the filter output obtained by examining a $V=1, H=2 D / 1.5$ portion of the grating. We then assume that the observer combines the $N=H V /(2 D / 1.5)$ estimates suboptimally so the SSNR increases by a factor of $N^{a}$, where $a$ is between $1 / 2$ and 1 , corresponding to some sort of imperfect predetection integration in the case of a radar receiver. ${ }^{27}$ We arbitrarily assume here the lowest value $a=1 / 2$. We compared all our results, assuming $a$ $=3 / 4$ as well, but could not choose between the two on the basis of our experiments. 


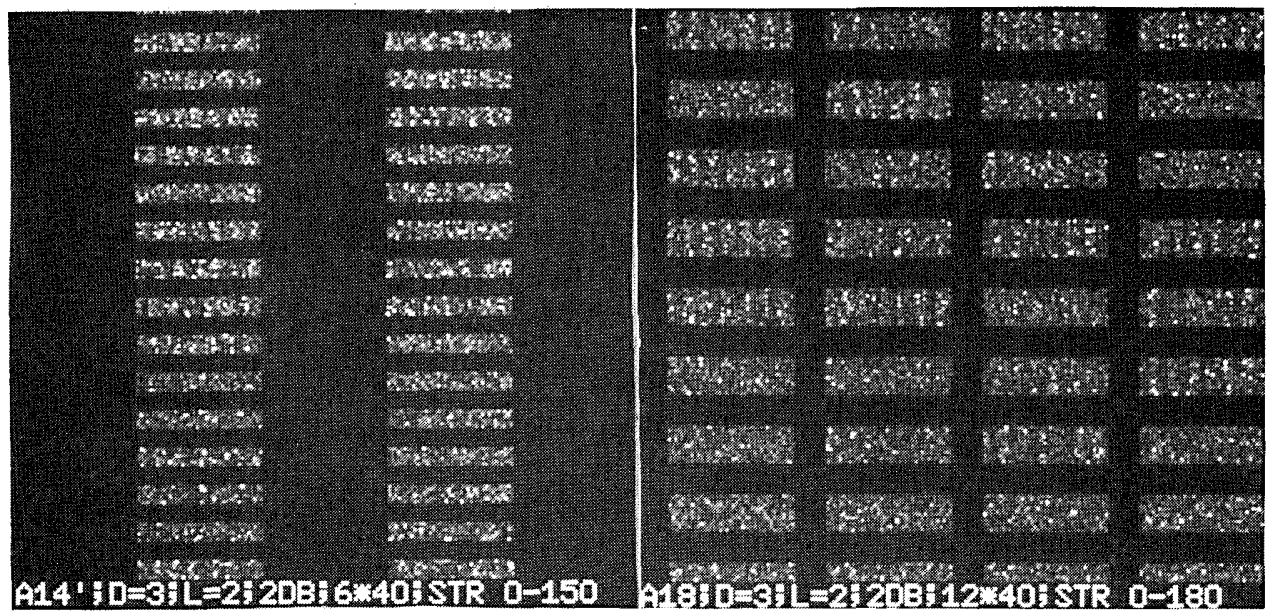

Fig. 4. Detection of gratings, examples of simulated pictures. Both photographs have $L=2, D=$ $3, H=40$, and 2-dB contrast: (left) $V=6$; (right) $V=12$.

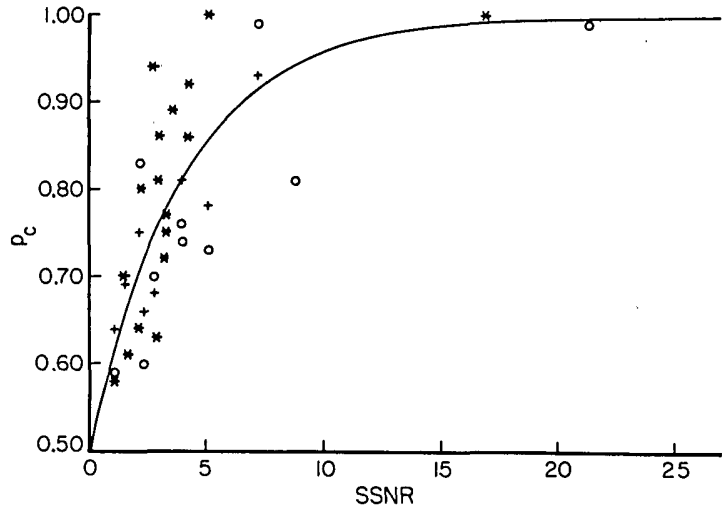

Fig. 5. Data points and regression curve: experimental $p_{c}$ vs SNNR: $\mathrm{O}$, data points with $V>H ;+$, data points with $V<H$; *, data points with $V=H$.

The expression for $\mathrm{SSNR}_{p}$ is obtained by finding ${ }^{17}$ approximately the signal and noise power outputs from the filter in one estimate and then multiplying $\mathrm{SSNR}_{p}$ by $N^{a}$ to get SSNR. The resulting expression is

$$
\begin{aligned}
\operatorname{SSNR} & =\frac{16}{\pi^{2}} \frac{(b / 2)^{2}}{(1+b / 2)^{2}+(b / 2)^{2}} \frac{2 D}{1.5} L\left(\frac{H V \cdot 1.5}{2 D}\right)^{1 / 2}, \\
& =\frac{16}{\pi^{2}} \frac{(b / 2)^{2}}{(1+b / 2)^{2}+(b / 2)^{2}} L\left(\frac{H V \cdot 2 D}{1.5}\right)^{1 / 2} .
\end{aligned}
$$

An interesting point in Eq. (9a) for the $\mathrm{SSNR}_{p}$ is the linear term in $D$; this is an agreement with the theory that the observer's eye sums horizontally over the width of each line, which we discussed in the feature detection problem.

\section{Simulations and Experiments}

Each picture simulated consisted of a matrix of $H \times$ $V$ patterns, each pattern randomly either noise or signal-plus-noise. The observer was previously shown an unspeckled grating with the same $D$ as the test grating and then asked to state whether each test pattern contained a grating (while all other patterns were covered by a mask). The experimental probability of correct decision was then obtained and plotted against the
SSNR expression. An exponential regression curve was then fitted to the data of $p_{c}$ vs SSNR; the resulting curve is

$$
\hat{p}_{c}=1-1 / 2 \exp [-(\mathrm{SSNR}) 0.25]+e,
$$

where $e$ is the error that is minimized in the least squared sense in obtaining the coefficient (0.25) multiplying SSNR in Eq. (10).

The index of correlation of the curve is 0.78 . Since this is fairly high, the experimentally observed $p_{c}$ is concluded to be well correlated with the calculated SSNR so that the SSNR expression defined is reasonable.

Figure 4 shows an example of the pictures simulated. The two photographs in Fig. 4 show the improvement in detectability of lines as $V$ increases from 6 to 12 , all other parameters being fixed $(L=2, D=3,2 \mathrm{~dB}, H=$ 40 ). Figure 5 shows the data points obtained from Table III, and the regression curve is fitted.

\section{E. Conclusions for Grating Detection}

(1) Figure 5 shows the points with $H>V$ and $H<$ $V$ plotted with different symbols. These two sets of points are visually seen to be similarly scattered, which indicates that an interchange of $H$ and $V$ should leave $p_{c}$ unchanged. (9).

This is also predicted by the SSNR expression of Eq.

(2) The SSNR expression indicates that an increase in $H$ or $V$ will increase $p_{c}$ so that a grating becomes more detectable as its size increases. This means that it is not sufficient to state the highest detectable grating frequency to characterize picture quality (as is commonly done): $H$ and $V$ also need to be specified.

\section{v. Comparison of Feature and Grating Detection}

We find from our regression curve that for $b=0.26$ (1-dB contrast), $L=12, D=2, H=V=100$, we have SSNR $=42.5$ and $p_{c}>0.999$.

From the feature detection calculations, we have that for $b=0.26, L=12,100 \times 100$ pictures, we need $D \simeq$ 7 to get $p_{c} \simeq 0.95$. 
By comparisons of this kind, we find that gratings with a given $D$ can be detected much more easily than small $D \times D$ squares in pictures with identical parameters. Figure 6 demonstrates this difference. In this figure, we have $D=6$ squares and $D=2$ lines in $100 \times$ 100 12-look 1-dB pictures. This shows that feature

Table ill. Line Pair Gratings

\begin{tabular}{rrrrrrrrr}
\hline No. & $(1+b)$ & $(1+b) \mathrm{dB}$ & $L$ & $D$ & $V$ & $H$ & $p_{c}$ & SSNR \\
\hline 1 & 1.26 & 1.0 & 1 & 2 & 64 & 64 & 0.80 & 2.21 \\
2 & 1.26 & 1.0 & 1 & 2 & 96 & 96 & 0.75 & 3.32 \\
3 & 1.26 & 1.0 & 1 & 1 & 120 & 120 & 0.63 & 2.93 \\
4 & 1.26 & 1.0 & 1 & 3 & 120 & 120 & 0.92 & 5.08 \\
5 & 1.26 & 1.0 & 1 & 3 & 100 & 100 & 0.89 & 4.24 \\
6 & 1.26 & 1.0 & 1 & 3 & 85 & 85 & 0.94 & 3.60 \\
7 & 1.26 & 1.0 & 1 & 3 & 64 & 64 & 0.64 & 2.71 \\
8 & 1.26 & 1.0 & 1 & 4 & 12 & 40 & 0.69 & 1.07 \\
9 & 1.26 & 1.0 & 1 & 4 & 24 & 40 & 0.75 & 1.52 \\
10 & 1.26 & 1.0 & 1 & 4 & 24 & 80 & 0.83 & 2.14 \\
11 & 1.26 & 1.0 & 1 & 4 & 80 & 24 & 0.81 & 2.14 \\
12 & 1.26 & 1.0 & 1 & 4 & 60 & 60 & 0.61 & 2.93 \\
13 & 1.26 & 1.0 & 12 & 2 & 4 & 4 & 0.66 & 1.66 \\
14 & 1.26 & 1.0 & 12 & 2 & 4 & 8 & 0.60 & 2.35 \\
15 & 1.26 & 1.0 & 12 & 2 & 8 & 4 & 0.77 & 2.35 \\
16 & 1.26 & 1.0 & 12 & 2 & 8 & 8 & 0.76 & 3.32 \\
17 & 1.60 & 2.0 & 3 & 2 & 12 & 8 & 0.72 & 3.93 \\
18 & 1.60 & 2.0 & 3 & 2 & 8 & 8 & 0.68 & 3.21 \\
19 & 1.60 & 2.0 & 3 & 2 & 6 & 8 & 0.70 & 2.78 \\
20 & 1.60 & 2.0 & 3 & 2 & 8 & 6 & 0.99 & 2.78 \\
21 & 1.60 & 2.0 & 2 & 3 & 40 & 12 & 0.93 & 7.18 \\
22 & 1.60 & 2.0 & 2 & 3 & 12 & 40 & 0.81 & 7.18 \\
23 & 1.60 & 2.0 & 2 & 3 & 120 & 6 & 0.73 & 8.80 \\
24 & 1.60 & 2.0 & 2 & 3 & 40 & 6 & 0.78 & 5.08 \\
25 & 1.60 & 2.0 & 2 & 3 & 6 & 40 & 0.74 & 5.08 \\
26 & 1.60 & 2.0 & 2 & 3 & 25 & 6 & 0.81 & 4.02 \\
27 & 1.60 & 2.0 & 2 & 3 & 6 & 24 & 0.59 & 3.93 \\
28 & 2.00 & 3.0 & 1 & 1 & 8 & 4 & 0.70 & 1.06 \\
29 & 2.00 & 3.0 & 1 & 1 & 8 & 8 & 0.86 & 1.50 \\
30 & 2.00 & 3.0 & 1 & 1 & 16 & 16 & 0.64 & 300 \\
31 & 2.00 & 3.0 & 1 & 2 & 8 & 8 & 0.58 & 2.12 \\
32 & 2.00 & 3.0 & 1 & 2 & 4 & 4 & 0.86 & 1.06 \\
33 & 2.00 & 3.0 & 1 & 2 & 16 & 16 & 0.99 & 4.24 \\
34 & 2.00 & 3.0 & 1 & 2 & 64 & 64 & 1.00 & 16.94 \\
35 & 3.16 & 5.0 & 2 & 3 & 40 & 6 & 1.00 & 21.33 \\
36 & 3.16 & 5.0 & 2 & 3 & 40 & 12 & 1.00 & 30.00 \\
\hline & & & & & & & &
\end{tabular}

detection rather than line pair detection ought to be used as a criterion for testing the quality of detectability provided by the overall system.

\section{Comparison with Previous Work}

Kozma and Christensen ${ }^{6}$ found that, if the contrast ratio of a grating was set at the value required to make $D=1$ lines just detectable in the absence of speckle, $D$ $=2.6$ would be required for the case of coherent illumination. Since we have not considered contrast ratios so low as to be just detectable in the absence of speckle, we cannot compare our results with theirs.

Dainty's calculations on small feature detection ${ }^{4}$ are based on a different detection mechanism. Since, in his calculations, the contrast ratio of the feature with respect to its surround appears only indirectly (through the feature and surround pdfs), we cannot directly compare our results with his. George et al. ${ }^{5}$ use firstorder statistics to relate contrast ratio to looks and consider just-detectable objects. Again, a direct numerical comparison with the results given here is not possible because the focus is on different points in George's paper so that some of the data required for such a comparison with our results are not given. However, as stated earlier, they have used and verified a relationship equivalent to our $M=L D^{2}$. We have not considered various cases, for instance, addition of a reference plane wave, as they do.

\section{Summary and Conclusions}

We have obtained plausible detection models for small feature detection and grating detection in speckled pictures and shown that experimental results support the theoretical predictions based on these models. Thus, we have given a method for calculating the probability of correct detection for these two problems, given the various parameters such as contrast, looks, and picture size. We have shown that if a test pattern is to be used to specify speckled picture quality,
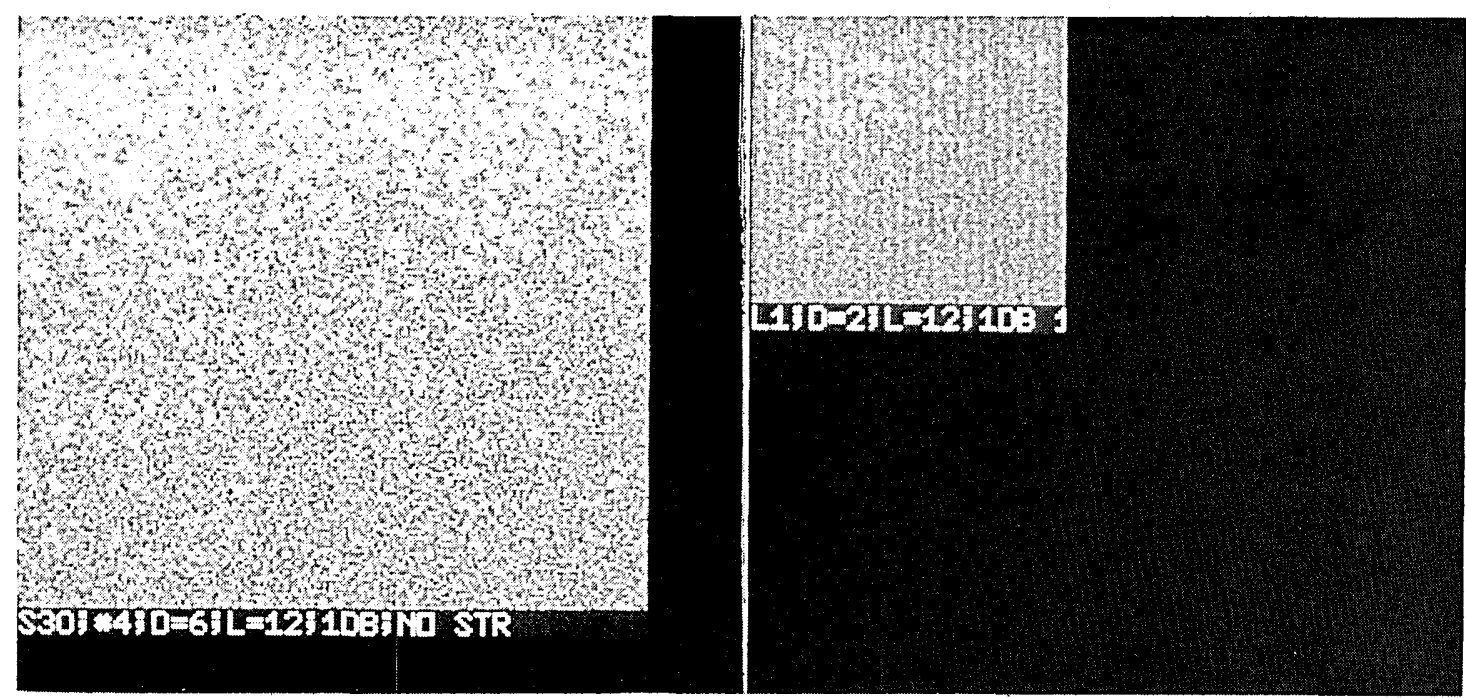

Fig. 6. Detectability of $D=6$ squares vs detectability of $D=2$ gratings in pictures with $L=12$ and 1 -dB contrast. There are four squares, one in each quadrant of the $200 \times 200$ photograph on the left; the photograph on the right is a $100 \times 100$ grating. 
small feature detectability, and not grating detectability, ought to be the criterion used. For this more important (feature detection) problem, we have given a useful formula for designing, for instance, the ratio (1 $+b)$ required to produce $95 \%$ detection probability of $D \times D$ squares in $W \times W L$-look pictures.

We would like to thank various members of the Jet Propulsion Laboratory, in particular, E. C. Posner and Chialin Wu for helpful discussions and R. G. Piereson for financial support and use of the facilities used in the simulations.

This work was carried out in part at the Jet Propulsion Laboratory, California Institute of Technology, Pasadena, under contract NAS7-100, sponsored by the National Aeronautics and Space Administration.

\section{References}

1. J. D. Rigden and E. I. Gordon, Proc. IRE 50, 2367 (1962).

2. J. W. Goodman, J. Opt. Soc. Am. 66, 1145 (1976).

3. J. C. Dainty, Ed., Laser Speckle and Related Phenomena (Springer, Berlin, 1975).

4. J. C. Dainty, Opt. Acta 18, 327 (1971).

5. N. George, C. R. Christensen, J. S. Bennett, and B. D. Guenther, J. Opt. Soc. Am. 66, 1282 (1976).

6. A. Kozma and C. R. Christensen, J. Opt. Soc. Am. 66, 1257 (1976).

7. R. D. Harger, Synthetic Aperture Radar Systems: Theory and Design (Academic, New York, 1970).

8. W. M. Brown, IEEE Trans. Aerosp. Electron. Syst. AES-3, 217 (1976).

9. L. J. Porcello, N. O. Massey, R. B. Innes, and J. M. Marks, J. Opt. Soc. Am. 6, 1305 (1976).

10. T. N. Cornsweet, Visual Perception (Academic, New York, 1970).

11. F. Radcliff, M. K. Hartline, and W. H. Miller, J. Opt. Soc. Am. 53, 110 (1963).

12. H. Nelson, J. Opt. Soc. Am. 53, 179 (1963).

13. L. D. Harmon and B. Julesz, Science 180, 1194 (1973).

14. D. M. Green and J. A. Swets, Signal Detection Theory and Psychophysics (Wiley, New York, 1966).

15. T. W. Bernard, A Symposium on Sampled Images (PerkinElmer, Norwalk, 1971).

16. J. M. Findlay, Vision Res. 9, 157 (1969).

17. V. N. Korwar, Ph.D. Thesis, California Institute of Technology, Pasadena (1980).

18. J. W. Goodman, Proc. IEEE 53, 1688 (1965).

19. E. J. Gumbel, Statistics of Extremes (Columbia U.P., New York, 1958).

20. G. S. Watson, Ann. Math. Stat. 25, 798 (1954).

21. M. Abramowitz and I. A. Stegun, Eds., Handbook of Mathematical Functions (U.S. GPO, Washington, D.C., 1970).

22. B. D. Guenther, C. R. Christensen, and A. Jain, at IEEE Computer Society Conference on Pattern Recognition and Image Processing, 78CH1318-5C (1978).

23. W. J. Dixon and F. J. Massey, Introduction to Statistical Analysis (McGraw-Hill, New York, 1969).

24. F. W. Campbell and J. G. Robson, J. Physiol. London 197, 551 (1968).

25. C. F. Stromeyer and B. Julesz, J. Opt. Soc. Am. 62, 1221 (1972).

26. R. M. Jones and U. Tulunay-Keesey, J. Opt. Soc. Am. 70, 66 (1980).

27. J. I. Marcum and P. Swerling, IRE Trans. Inf. Theory IT-6, 59 (1960).
Meetings Calendar continued from page 306

1981

April

8-10 2nd Int. Conf. on Distributed Computing Systems, Paris Institut National de Recherche en Informatique et en Automatique, Domaine de Voluceau-Rocquencourt, 78150 Le Chesnay, France

13-15 Miami Int. Symp. on Metal-Hydrogen Systems, Miami Beach T. Veziroglu, Clean Energy Res. Inst., $U$. Miami, P.O. Box 248294, Coral Gables, Fla. 33124

13-17 Measurements of Laser Output Characteristics course Denver, Colo. Laser Inst. Am., P.O. Box 9000, Waco, Tex. 76710

14-18 SEM/1981, Dallas O. Johari, SEM Inc., P.O. Box 66507, AMF O'Hare, Ill., 60666

20-24 SPIE Technical Symposium East '81, Wash., D.C. SPIE, P.O. Box 10, Bellingham, Wash. 98227

26-1 May Freeze Etching in Electron Microscopy course, Woods Hole, Mass. Marine Biological Lab., Woods Hole, Mass. 02543

27-29 Integrated Optics and Optical Fiber Communication, 3rd Int. Conf., San Francisco OSA, 1816 Jefferson $\mathrm{Pl}$. N.W., Wash., D.C. 20036

28-30 EFOC 81, 2nd European Fiber Optics and Communications Expo., Paris E. Bond, Info. Gatekeepers, Inc., 167 Corey Rd., Suite 111, Brookline, Mass. 02146

28-30 Smoke/Obscurants Symp. V, Adelphi, Md. Smoke/ Obscurants, U.S. Army, DRCPM-SMK-T, Aberdeen Proving Ground, Md. 21005

30-1 May 6th Conf. Roofing Technology, NBS, Gaithersburg $R$. Mathey, B-348 Bldg. Tech., NBS, Wash., D.C. 20234

30-1 May 12th Ann. Pittsburgh Conf. Modeling and Simulation, Pittsburgh W. Vogt, Modeling \& Simulation Conf., 348 Benedum Eng. Hall, Pittsburgh U., Pittsburgh, Pa. 15261

May

3-5 Electron Microscopy in the Biological Sciences course, Woods Hole, Mass. Marine Biological Lab., Woods Hole, Mass. 02543

4-6 Gradient Index Optical Imaging Systems, OSA Topical Mtg., Honolulu OSA, 1816 Jefferson Pl. N.W., Wash., D.C. 20036

6-7 Laser-Optics 3, Andover, Mass. M. Fournier, U. Lowell, Cont. Ed., 1 University Ave., Lowell, Mass. 01854

10-14 34th SPSE Ann. Conf., N.Y. R. Wood, SPSE, Central P.O. Box 28327, Wash., D.C. 20005

10-16 Analytical and Quantitative Light Microscopy in Biology, Medicine, and Materials Sciences course, Woods Hole, Mass. Marine Biological Lab., Woods Hole, Mass. 02543

continued on page 325 